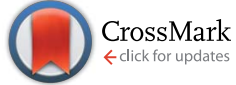

Cite this: J. Mater. Chem. A, 2016, 4, 15162

Received 12th June 2016

Accepted 1st September 2016

DOI: $10.1039 / c 6 t a 04917 f$

www.rsc.org/MaterialsA

\section{Anionic porous polymers with tunable structures and catalytic properties $\dagger$}

\author{
Wuxue Zhao, ${ }^{a}$ Fan Zhang, ${ }^{\text {a }}$ Lingyun Yang, ${ }^{\mathrm{b}}$ Shuai $\mathrm{Bi}^{\mathrm{a}}{ }^{\mathrm{a}}$ Dongqing $\mathrm{Wu}{ }^{\mathrm{a}}$ Yefeng $\mathrm{Yao},{ }^{\mathrm{b}}$ \\ Manfred Wagner, ${ }^{c}$ Robert Graf, ${ }^{c}$ Michael Ryan Hansen, ${ }^{\text {cd }}$ Xiaodong Zhuang*ae \\ and Xinliang Feng ${ }^{\text {ae }}$
}

A series of boron-containing conjugated microporous polymers with hierarchical porous structures have been readily prepared via typical transition metal-catalyzed coupling reactions. The distribution of microand mesopores in the networks as well as the specific surface areas are tunable via tailoring the structures of the building blocks. The distinct capability of the resulting Lewis acid-based neutral porous polymers to selectively capture fluoride ions provides a high-efficiency conversion into stable anionic porous polymers. For the first time, fluoride anion binding to boron atoms in a solid sample was essentially characterized by solid-state ${ }^{11}$ B MAS NMR spectroscopy, clearly revealing such an efficient conversion from a neutral network to a negatively charged one only through Lewis acid-base adduct formation. Upon a simple ion-exchange process, various heavy metal cations were facile to be loaded into the networks of the anionic porous polymers. Furthermore, the cobalt(I)-loaded porous polymers were shown to promote the stoichiometric homocoupling reactions of the different aryl Grignard regents, and exert distinct size selectivities for the homocoupling products, highly dependent on their porous structures. Such a successful loading strategy might be used for design and synthesis of new types of zeolite-like porous polymers with desirable catalytic properties for a certain organic transformation, as well as other functional materials.

\section{Introduction}

Porous materials ${ }^{\mathbf{1}}$ have attracted tremendous academic and commercial attention in numerous fields, such as membrane separation, ${ }^{2}$ industrial catalysis, ${ }^{3}$ and energy conversion and storage. ${ }^{4}$ Among them are metal organic frameworks (MOFs), ${ }^{5}$ consisting of organic linkers and coordinated metal centers, that have cationic networks with extra-framework counter anions. In contrast, zeolites and $\mathrm{MOFs}^{6}$ possess anionic networks balanced with extra-network counter-cations to maintain the electroneutrality of the materials. The unique structural and porous features of zeolites facilitate their use as

${ }^{a}$ School of Chemistry and Chemical Engineering, State Key Laboratory of Metal Matrix Composites, Shanghai Key Laboratory of Electrical Insulation and Thermal Ageing, Shanghai Jiao Tong University, 800 Dongchuan Road, Shanghai 200240, China. E-mail: fan-zhang@sjtu.edu.cn; zhuang@sjtu.edu.cn

${ }^{b}$ Physics Department \& Shanghai Key Laboratory of Magnetic Resonance, East China Normal University, North Zhongshan Road 3663, 200062 Shanghai, China

'Max Planck Institute for Polymer Research, Ackermannweg 10, D-55128 Mainz, Germany

${ }^{d}$ Institute of Physical Chemistry, Westfälische Wilhelms-Universität Münster, Corrensstr. 28/30, D-48149 Münster, Germany

${ }^{e}$ Center for Advancing Electronics Dresden (cfaed), Department of Chemistry and Food Chemistry, Technische Universitaet Dresden, 01062 Dresden, Germany

$\dagger$ Electronic supplementary information (ESI) available. See DOI: $10.1039 /$ c6ta04917f heterogeneous catalysts in many organic transformations, such as Friedel-Crafts alkylation, ${ }^{7}$ alkylaromatic isomerization ${ }^{8}$ and disproportionation, ${ }^{9}$ realized on an industrial scale. However, due to the lack of controlled and versatile functionalization at the molecular level, the structures of zeolites cannot be finely tailored. A lot of work remains to be done to understand the relationship between the catalytic mechanism and a material's structure. $^{\mathbf{1 0}}$

Porous polymer networks are generally built up from light elements, such as carbon, nitrogen, boron and oxygen, and are formed by cross-linking organic-directing and building units through covalent bonds. ${ }^{11}$ Their well-defined structure can be tailored at the molecular level to optimize their textural and chemical properties. Compared with MOFs, porous organic polymers exhibit much higher chemical and thermal stabilities. ${ }^{12}$ In general, porous polymers feature a neutral character, but in the past few years, some cationic porous polymers have been reported, ${ }^{13}$ while the anionic porous polymers remain rarely explored. Very recently, anionic microporous polymers were prepared by using tetraaryl boron anion units, suitable for being used as catalysts, solid electrolytes or absorbents, thus arousing much more attention to the charged porous polymers. ${ }^{14}$

In this paper, we present a concise synthetic strategy toward a series of neutral Lewis acid boron-containing porous 
polymers, which can be readily converted into anionic porous polymers via Lewis acid-base interactions with fluoride ions. Further, various alkali or heavy metals can be selectively loaded on such anionic porous polymers by a simple ion-exchange process, representing the first example of the successful loading approach to functional porous materials. The as-prepared neutral and anionic porous polymers exhibit high permanent surface areas and hierarchical porous structures. Remarkably, as an example, the cobalt(II)-loaded porous polymers can effectively promote stoichiometric homocoupling reactions of aryl Grignard regents with molecular size selectivity depending on the porous features of the networks.

\section{Experimental section}

All starting materials were purchased from Aladdin and Aldrich. All air-sensitive reactions were carried out under a nitrogen atmosphere and performed using Schlenk techniques. All solvents were dried before use. Tetrahydrofuran (THF) was refluxed with sodium. Triethylamine (TEA) and dichloromethane were refluxed with calcium hydroxide. Tris(bromoduryl)borane, ${ }^{15}$ 4,4-diethynyl-1,1-biphenyl, ${ }^{16}$ 1,4-diethynylbenzene, ${ }^{16}$ and $1,3,5$ triethynylbenzene, ${ }^{17}$ were synthesized according to the reported procedures.

\section{General protocol for the synthesis of neutral B-Ph-ae-n}

All polymerization reactions were carried out at $75{ }^{\circ} \mathrm{C}$ for $72 \mathrm{~h}$. The molar ratio of ethynyl to bromo functionalities in the monomer feed was set at $1.5: 1$. The typical experimental procedure is given as follows:

Synthesis of B-Ph-ae-1. 1,4-Diethynylbenzene (219 mg, 1.74 $\mathrm{mmol}$ ), tris(bromoduryl)borane $(500 \mathrm{mg}, 0.77 \mathrm{mmol})$, (triphenylphosphine) palladium (200 $\mathrm{mg}, 0.174 \mathrm{mmol}$ ), and copper(I) iodide (34.0 mg, $0.174 \mathrm{mmol}$ ) were added into a mixture of anhydrous $\mathrm{THF}(15 \mathrm{~mL})$ and $\mathrm{Et}_{3} \mathrm{~N}(10 \mathrm{~mL})$. The reaction mixture was heated to $75{ }^{\circ} \mathrm{C}$ and stirred for $72 \mathrm{~h}$ under a nitrogen atmosphere. Afterwards, the mixture was cooled to room temperature, and the resulting precipitate was collected by filtration and washed sequentially with chloroform, water, ethanol, and acetone to remove any un-reacted monomers or catalyst residues, and further purified by Soxhlet extraction with methanol for $24 \mathrm{~h}$. Finally, the product was dried under vacuum for $24 \mathrm{~h}$ at $60{ }^{\circ} \mathrm{C}$ to afford the porous polymer B-Ph-ae-1 as a yellow powder $(512 \mathrm{mg}, 87 \%$ yield $)$.

Synthesis of B-Ph-ae-2. Starting from 4,4-diethynyl-1,1biphenyl (352 mg, $1.74 \mathrm{mmol}$ ), tris(bromoduryl)borane (500 mg, $0.77 \mathrm{mmol}$ ), (triphenylphosphine)palladium (200 mg, 0.174 $\mathrm{mmol}$ ), and copper(I) iodide (34.0 $\mathrm{mg}, 0.174 \mathrm{mmol})$ were added into a mixture of THF $(15 \mathrm{~mL})$ and $\mathrm{Et}_{3} \mathrm{~N}(10 \mathrm{~mL})$. Porous polymer B-Ph-ae-2 was obtained as a yellow powder (634 mg, 88\% yield) via the same procedure as described above.

Synthesis of B-Ph-ae-3. Starting from 1,3,5-triethynylbenzene (174 mg, $1.16 \mathrm{mmol}$ ), tris(bromoduryl)borane (500 mg, 0.77 mmol), (triphenylphosphine)palladium (200 mg, $0.174 \mathrm{mmol}$ ), and copper(I) iodide (34.0 $\mathrm{mg}, 0.174 \mathrm{mmol}$ ) were added into a mixture of $\mathrm{THF}(15 \mathrm{~mL})$ and $\mathrm{Et}_{3} \mathrm{~N}(10 \mathrm{~mL})$. Porous polymer
B-Ph-ae-3 was obtained as yellow powder (522 $\mathrm{mg}$, 91\% yield) via the same procedure as described above.

\section{General protocol for the synthesis of anionic porous polymers MF@B-Ph-ae-n}

Synthesis of anionic porous polymer F@B-Ph-ae-n. The neutral polymer B-Ph-ae- $\boldsymbol{n}$ was added into a solution of thiocarbamide and stirred for $2 \mathrm{~h}$ at $80{ }^{\circ} \mathrm{C}$ in order to remove the palladium catalyst. Then, B-Ph-ae- $\boldsymbol{n}$ and an excess amount of tetrabutylammonium fluoride $\left(\mathrm{Bu}_{4} \mathrm{NF}\right)$ were mixed in anhydrous THF and stirred for $12 \mathrm{~h}$. After centrifugation, the precipitate was collected, and stirred in a fresh anhydrous THF solution of $\mathrm{Bu}_{4} \mathrm{NF}$ again for $12 \mathrm{~h}$. Finally, the resulting precipitate was collected, washed with THF, and dried for $1 \mathrm{~h}$ under high vacuum, affording the F@B-Ph-ae- $\boldsymbol{n}$.

Synthesis of heavy metal-loaded anionic porous polymer MF@B-Ph-ae-n. The ion exchange was carried out by treatment of F@B-Ph-ae- $\boldsymbol{n}$ with an excess amount of one or several $\mathrm{M}(\mathrm{Ac})_{2}$ salts (M: Co, $\mathrm{Fe}, \mathrm{Cu}$, and $\mathrm{Ni}$ ) in a solvent mixture of ethanol and THF, for 2 days, and then centrifuged. The resulting solid powder was collected, washed with ethanol several times and dried under high vacuum, affording the MF@B-Ph-ae-n.

\section{Results and discussion}

The synthetic route toward Lewis acid boron-based porous polymers B-Ph-ae- $\boldsymbol{n}(n=1,2$, and 3$)$ is presented in Scheme 1. The key monomers of tris(bromoduryl)borane (1), 1,4-diethynylbenzene (2), 4,4-diethynyl-1,1-biphenyl (3), and 1,3,5-triethynylbenzene (4) were synthesized according to previous reports (see the ESI $\dagger$ ). Under a typical Sonogashira crosscoupling condition, monomer 1 was reacted with monomers $\mathbf{2 - 4}$, to afford the porous polymers B-Ph-ae-1, B-Ph-ae-2 and B-Ph-ae-3, all as yellow powders in high yields (from $87 \%$ to

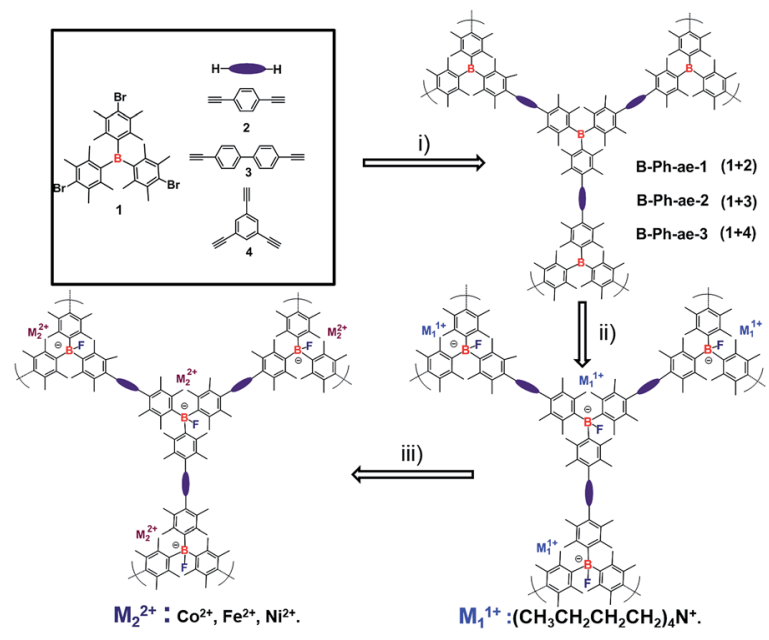

Scheme 1 Synthesis of Lewis acid-based neutral porous polymers and corresponding ionic porous polymers. (i) $\mathrm{Et}_{3} \mathrm{~N}, \mathrm{Pd}\left(\mathrm{PPh}_{3}\right)_{4}, \mathrm{Cul}, 75^{\circ} \mathrm{C}$, $72 \mathrm{~h}$, tetrahydrofuran. (ii) Tetrabutylammonium fluoride $\left(\mathrm{Bu}_{4} \mathrm{NF}\right), \mathrm{THF}$, room temperature (RT). (iii) Ethanol and THF, cobalt acetate, nickel acetate, and iron nitrate, at RT. 
91\%). The as-synthesized porous polymers were revealed to exhibit excellent stabilities after treatment with water (Fig. S1 $\dagger$ ). The duryl moiety containing four sterically bulky methyl groups at the 2, 3, 5, and 6-positions of the phenyl ring in monomer 1 is clearly beneficial for achieving high polymerization efficiency as well as to improve the inertness of the resulting porous polymers to moisture and oxygen.

The structures of the polymeric networks B-Ph-ae-1, B-Ph-ae2, and B-Ph-ae-3 were characterized by Fourier transform infrared (FTIR) spectroscopy (Fig. S2a $\dagger$ ) and ${ }^{13} \mathrm{C}$ solid-state nuclear magnetic resonance (NMR) spectroscopy (Fig. 1a). In the FTIR spectra, a typical C-B stretching signal at around 1105 $\mathrm{cm}^{-1}$ was observed for all three porous polymers. A peak with a very low intensity at $2110 \mathrm{~cm}^{-1}$ corresponds to $-\mathrm{C}=\mathrm{C}$ stretching. The broad peaks at $3300 \mathrm{~cm}^{-1}$ can be attributed to the $-\mathrm{C}=\mathrm{C}-$ linkages. The signals at $2870-3050 \mathrm{~cm}^{-1}$ originate from aromatic $\mathrm{C}-\mathrm{H}$ stretching. ${ }^{18}$ In the solid-state ${ }^{13} \mathrm{C}\left\{{ }^{1} \mathrm{H}\right\}$ cross-polarization/magic angle spinning (CP/MAS) NMR spectra, the well-resolved resonances at around $20.0 \mathrm{ppm}$ are ascribed to the methyl carbons $\left(\mathrm{C}_{\mathrm{ar}}-\mathrm{CH}_{3}\right)$. The peaks at $\sim 136.3$ and $131.0 \mathrm{ppm}$ can be attributed to the substituted aromatic carbons $\left(\mathrm{C}_{\mathrm{ar}}-\mathrm{CH}_{3}\right)$ and the hydrogen-bearing aromatic carbons $\left(C_{\mathrm{ar}}-\mathrm{H}\right)$, respectively. The signals located at $\sim 123.3$ and 91.6 ppm can be assigned to $\left(C_{\mathrm{ar}}-\mathrm{C}=\mathrm{C}-C_{\mathrm{ar}^{\prime}}\right)\left(C_{\mathrm{ar}}\right.$ and $C_{\mathrm{ar}^{\prime}}$ are the carbon atoms of duryl and phenyl rings, respectively; they cannot be distinguished under the current measurement conditions) and the carbon atoms of the quaternary alkynes $\left(\mathrm{C}_{\mathrm{ar}}-\mathrm{C}=C-\mathrm{C}_{\mathrm{ar}}\right)$, respectively. There are no detectable resonances at about 83.0 and $77.0 \mathrm{ppm}$ from the terminal acetylenic groups $\left(\mathrm{C}_{\mathrm{ar}}-\mathrm{C}=C-\mathrm{H}\right)$, suggesting a high polymerization efficiency for the porous polymers. ${ }^{19}$

$\mathrm{X}$-ray diffraction (XRD) (Fig. S2b $\dagger$ ) demonstrates the amorphous features that are typical for porous polymers built up

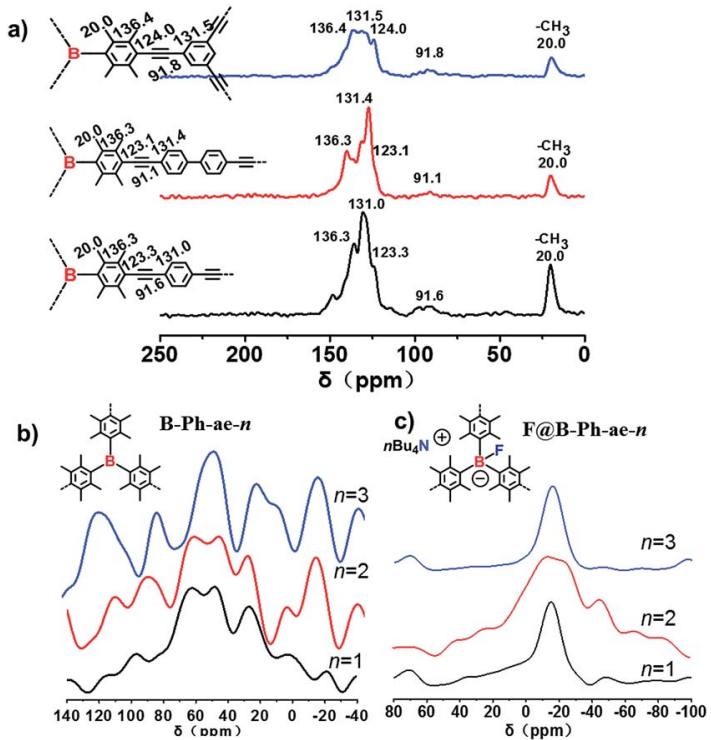

Fig. 1 (a) Solid-state ${ }^{13}$ CCP/MAS NMR spectra of B-Ph-ae-1 (black), BPh-ae-2 (red), and B-Ph-ae-3 (blue); (b) solid-state ${ }^{11} \mathrm{~B}$ MAS NMR spectra of B-Ph-ae- $n$; (c) solid-state ${ }^{11} \mathrm{~B}$ MAS NMR spectra of F(B-Phae- $n$. from metal-catalyzed coupling reactions. Thermogravimetric analysis (TGA) (Fig. S2c $\dagger$ ) under a nitrogen atmosphere manifests a weight loss of only $5 \%$ at 315, 312 and $267^{\circ} \mathrm{C}$ for B-Ph-ae1, B-Ph-ae-2, and B-Ph-ae-3, respectively, suggesting good thermal stability for these polymers. The morphology and microstructure of the polymer networks were investigated by transmission electron microscopy (TEM) (Fig. S3†). The TEM images of polymers show the presence of a vermicular texture. The alternating areas of light and dark contrast in the TEM images disclose a disordered porous structure for the porous polymers.

The porous nature of the polymer networks was further confirmed by nitrogen physisorption measurements. It was found that these porous polymers exhibit type IV isotherms. ${ }^{20}$ The distinct hysteresis phenomena between the adsorption and desorption cycles at relatively low pressure for $\mathbf{B}-\mathbf{P h}-\mathbf{a e - 1}$, and, in particular, for $\mathbf{B}-\mathbf{P h}-\mathbf{a e - 2}$, stem from the deformation and swelling of the polymer networks. ${ }^{21}$ The Brunauer-EmmettTeller (BET) surface areas of these polymer networks increased in the sequence of B-Ph-ae-2 $\left(226 \mathrm{~m}^{2} \mathrm{~g}^{-1}\right)<$ B-Ph-ae-1 $\left(432 \mathrm{~m}^{2}\right.$ $\left.\mathrm{g}^{-1}\right)<$ B-Ph-ae-3 $\left(935 \mathrm{~m}^{2} \mathrm{~g}^{-1}\right)$. The pore size distribution curves for these polymers from the adsorption branches of the isotherms were calculated using non-local density functional theory (NL-DFT) (Fig. 2). A set of peaks from 0.88 to $1.94 \mathrm{~nm}$ are found for B-Ph-ae-2, demonstrating its distinct microporous features. However, two main peaks at 1.62 and $3.85 \mathrm{~nm}$ for B-Phae-1 and three narrowly distributed peaks with maxima at 1.70, 2.70, and $3.90 \mathrm{~nm}$ for B-Ph-ae-3 were observed, signifying the presence of both micro- and meso-pores in the networks. This
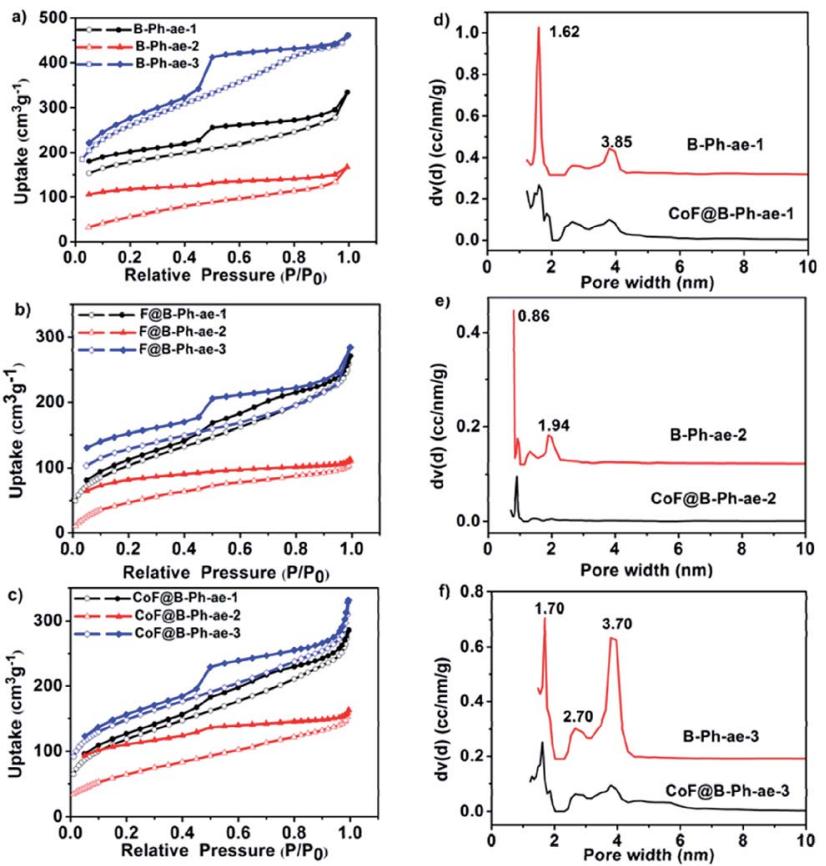

Fig. 2 Nitrogen adsorption (open symbols) and desorption (solid symbols) isotherms of (a) B-Ph-ae- $n$, (b) FaB-Ph-ae- $n$, and (c) CoFaB-Ph-ae- $n$ measured at $77 \mathrm{~K}$. Pore size distribution curves of (d) B-Ph-ae-1 and CoFaB-Ph-ae-1, (e) B-Ph-ae-2 and CoFaB-Ph-ae-2, and (f) B-Ph-ae-3 and CoF@B-Ph-ae-3 as calculated by NL-DFT. 
result is in good agreement with the profiles of $\mathrm{N}_{2}$ absorption isotherms. Compared with B-Ph-ae-3, B-Ph-ae-2 is constructed from the conjugated monomer with a longer chain length, and B-Ph-ae-1 exhibits a lower surface area and smaller pore sizes, which can be ascribed to the possible formation of an interpenetrating network and a coiled structure as the backbone rigidity decreased.

Given that the Lewis-acid boron center in tridurylborane has a highly selective and strong binding capacity for $\mathrm{F}^{-},{ }^{22}$ we consider that the as-prepared neutral porous polymers can be readily converted into corresponding anionic porous polymers, as shown in Scheme 1. Thus, the neutral polymers B-Ph-ae- $\boldsymbol{n}(n$ $=1,2$, and 3) were suspended in the THF solution of tetrabutylammonium fluoride $\left(\mathrm{Bu}_{4} \mathrm{NF}\right)$, and stirred at room temperature for 2 days to afford the anionic porous polymers F@B-Phae- $\boldsymbol{n}(n=1,2$, and 3$)$, respectively.

In order to reveal the difference between the samples before and after $n \mathbf{B u}_{4} \mathrm{NF}$ treatment, B-Ph-ae- $\boldsymbol{n}$ and F@B-Ph-ae- $\boldsymbol{n}$ were studied by X-ray photoelectron spectroscopy (XPS). For the neutral B-Ph-ae-n, all XPS spectra of these samples show a B1s region of $188.4 \mathrm{eV}$, which can be attributed to typical B-C bonds (Fig. S4 †). After ionization, two B1s peaks at 190.3 and $188.4 \mathrm{eV}$ can be observed for F@B-Ph-ae- $\boldsymbol{n},{ }^{23}$ which can be ascribed to BF and B-C bonds, respectively (Fig. S5 †). B-Ph-ae- $\boldsymbol{n}$ and F@B-Phae- $\boldsymbol{n}$ were further characterized by ${ }^{11} \mathrm{~B}$ solid-state nuclear magnetic resonance (SS-NMR) spectroscopy (see Fig. 1b and c). From the ${ }^{11} B$ MAS NMR spectra of B-Ph-ae- $\boldsymbol{n}$, the broad signal centered at around $55 \mathrm{ppm}$ can be assigned to B incorporated into the conjugated polymer networks at regular positions located in symmetric environments. ${ }^{11 d}$ As a contrast, in the ${ }^{11} \mathrm{~B}$ MAS NMR spectra of F@B-Ph-ae-n (Fig. 1c), a new relatively sharp signal at $-15.8 \mathrm{ppm}$ in the high field regions can be observed and the peak at around $55 \mathrm{ppm}$ almost disappeared in the case of F@B-Ph-ae-3, essentially suggestive of approximately all boron atoms coordinated with $\mathrm{F}^{-}$in the network. This result coincides well with the ${ }^{11} \mathrm{~B}$ NMR chemical shift of the model compound (Fig. S6 $†$ ). Notably, for F@B-Ph-ae-1 or F@B-Ph-ae-2, the main signal at $-15.8 \mathrm{ppm}$, with the tailed peaks in the low field regions, might be attributed to the presence of no $\mathrm{F}^{-}$binding boron atoms. Such a phenomenon is highly related to the porous structure character in the different porous polymer samples. Furthermore, the binding of $\mathrm{F}^{-}$to boron atoms in the porous polymers and model compound was also verified by ${ }^{19} \mathrm{~F}$ NMR spectroscopy (Fig. S7 †). The signal at $-148.8 \mathrm{ppm}$ can be clearly attributed to $\mathrm{F}$ atoms in F-B bonds, highly in line with the ${ }^{19} \mathrm{~F}$ NMR chemical shift of the model compound (Fig. S8 ${ }^{\dagger}$ ) and the reported literature. ${ }^{24}$ All these characterizations strongly indicate that the ionic porous polymers F@B-Ph-ae-n have been successfully synthesized by the simple treatment of the neutral porous polymers B-Ph-ae- $\boldsymbol{n}$ with $n \mathrm{Bu}_{4} \mathrm{NF}$ in some common organic solvents.

The loading degree of $\mathrm{F}^{-}$was next examined by fluorescence spectroscopy. A decreased intensity of the fluorescence emission was found (Fig. S9†), provided the residual empty orbitals of boron atoms in the polymer networks were gradually occupied by the fluoride anions. ${ }^{25}$ The very low dissociation constant of the $\mathrm{F}^{-}$bonded triduryl boron moiety ${ }^{26}$ essentially ensures that the framework consisting of such a kind of moiety is able to be kept in a relatively stable charged state. The content of $\mathrm{F}^{-}$ remained unchanged after washing many times with THF for thoroughly removing off the free $n \mathrm{Bu}_{4} \mathrm{NF}$. Furthermore, considering that the exact content of fluoride in a sample is normally difficult to be determined directly by elemental analysis, ${ }^{27}$ alternatively, we can measure the content of nitrogen (from the counter cation tetrabutyl ammonium ions, equal molar amount relative to the fluoride binding to boron) in the as-prepared F@B-Ph-ae- $\boldsymbol{n}$ samples. As an example, F@B-Ph-ae-3 was examined to have a content of $\mathrm{N}(1.52 \mathrm{wt} \%)$; thus the content of $\mathrm{F}(2.05 \mathrm{wt} \%)$ can be evaluated. Meanwhile, by comparing with the content of B (1.48 wt\%) from elemental analysis, we can confirm that $\mathrm{F}$ and $\mathrm{B}$ exist nearly in a $1: 1$ molar ratio in F@B-Ph-ae-3. The detailed B, N and F contents of F@BPh-ae- $\boldsymbol{n}$ are summarized in Table S1. $\dagger$ After ionization, the surface areas of F@B-Ph-ae- $n$ decreased by $10.4 \%(n=1), 22.6 \%$ $(n=2)$ and $54.7 \%(n=3)$ in comparison with those of B-Ph-ae- $\boldsymbol{n}$. This result can be easily attributed to the increased weight after ionization. The relatively poor binding performance of B-Ph-ae$\mathbf{1}$ and B-Ph-ae-2 can be attributed to their lower surface area and higher ratio microporous structural characters. These results are well in agreement with the ${ }^{11} \mathrm{~B}$ NMR spectra analyses above. The $\mathrm{N}_{2}$ adsorption isotherm manifested significantly the reduced surface areas and pore volumes for the anionic F@BPh-ae- $\boldsymbol{n}$ compared with the neutral B-Ph-ae- $\boldsymbol{n}$ (Table 1). This can be explained by the increased molecular mass and space filling resulting from the loaded potassium ions. Interestingly, the pore volume of $0.62 \mathrm{~cm}^{3} \mathrm{~g}^{-1}$ for F@B-Ph-ae-3 is much higher than that of $0.48 \mathrm{~cm}^{3} \mathrm{~g}^{-1}$ for recently reported anionic porous polymers with the counter cation $\mathrm{Na}^{+} \cdot{ }^{14}$

Ion exchange is a favorable strategy to incorporate various ions into the charged materials, e.g. zeolites, for achieving the desired properties, such as catalysis ${ }^{28}$ and gas adsorption. ${ }^{13,29} \mathrm{As}$ expected, the tetrabutyl ammonium cations in the networks of the as-prepared F@B-Ph-ae- $\boldsymbol{n}$ can be readily exchanged with the different heavy metal ions by the simple treatment with the corresponding heavy metal salt in a solvent mixture of ethanol and THF, resulting in heavy metal ion loaded porous polymers

Table 1 Physical properties of B-Ph-ae- $n$, F@B-Ph-ae- $n$, and CoF@BPh-ae- $n$ polymer networks

\begin{tabular}{llll}
\hline Sample & $S_{\text {BET }}{ }^{a}\left(\mathrm{~m}^{2} \mathrm{~g}^{-1}\right)$ & $V_{\text {micro }}{ }^{b}\left(\mathrm{~cm}^{3} \mathrm{~g}^{-1}\right)$ & $V_{\text {tot }}{ }^{c}\left(\mathrm{~cm}^{3} \mathrm{~g}^{-1}\right)$ \\
\hline B-Ph-ae-1 & 432 & 0.14 & 0.26 \\
B-Ph-ae-2 & 226 & 0.12 & 0.42 \\
B-Ph-ae-3 & 935 & 0.20 & 0.71 \\
F@B-Ph-ae-1 & 387 & 0.10 & 0.15 \\
F@B-Ph-ae-2 & 175 & 0.08 & 0.22 \\
F@B-Ph-ae-3 & 424 & 0.13 & 0.47 \\
CoF@B-Ph-ae-1 & 424 & 0.11 & 0.17 \\
CoF@B-Ph-ae-2 & 186 & 0.10 & 0.26 \\
CoF@B-Ph-ae-3 & 490 & 0.15 & 0.53
\end{tabular}

${ }^{a}$ Surface areas calculated from the $\mathrm{N}_{2}$ adsorption isotherm using the BET method. ${ }^{b}$ The micropore volume derived using the non-local density functional theory. ${ }^{c}$ Total pore volume at $p / p_{0}=0.99$. 
MF@B-Ph-ae-n (see the ESI $\dagger$ ). Here, CoF@B-Ph-ae-3 was selected as a typical example for the detailed investigation. According to ICP-OES analysis, the loading content of cobalt in Co@B-Ph-ae-3 is $2.43 \mathrm{wt} \%$, which is nearly half the molar amount of $\mathrm{B}(1.11 \mathrm{wt} \%)$ in the corresponding F@B-Ph-ae-3 sample, suggesting that tetrabutyl ammonium ions have been almost completely replaced by $\mathrm{Co}(\mathrm{II})$ ions to maintain the charge balance. In addition, the acetate anion signal was not observed in the FTIR spectra (Fig. S10†), confirming that there is no free $\mathrm{Co}(\mathrm{Ac})_{2}$ left in the networks.

The compositions of the resulting Co(II) loaded anionic polymers were further analyzed via X-ray photoelectron spectroscopy (XPS) (Fig. 3 and S11, S12 $\dagger$ ). There are two characteristic peaks at 782.2 and $798.5 \mathrm{eV}$, corresponding to the $2 \mathrm{p} 3 / 2$ and $2 \mathrm{p} 1 / 2$ spin-orbit peaks of $\mathrm{Co}$ (II). The peaks at around 787.2 and $803.3 \mathrm{eV}$ are associated with the shake-up type peaks of the $2 \mathrm{p} 3 / 2$ and 2 p1/2 edges, respectively. ${ }^{30}$ XPS also indicates a cobalt content of $2.40 \mathrm{wt} \%$, which is in good agreement with the value obtained from ICP-OES analysis. Of note, elemental mapping images show the homogeneous distribution of carbon, boron, and cobalt elements in CoF@B-Ph-ae-3 (Fig. 3). The surface area and pore volume of CoF@B-Ph-ae-3 were determined to be $468 \mathrm{~m}^{2} \mathrm{~g}^{-1}$ and $0.51 \mathrm{~cm}^{3} \mathrm{~g}^{-1}$, respectively, which are slightly lower than those of F@B-Ph-ae-3 (Table 1) due to the lower molecular weight of $\mathrm{Co}^{2+}$ (58.93) in comparison with $\mathrm{Bu}_{4} \mathrm{~N}^{+}$ (242.28). Therefore, one can recognize the remarkable differences in $\mathrm{N}_{2}$ isotherm profiles, surface areas, and pore volumes for B-Ph-ae- $\boldsymbol{n}$, F@B-Ph-ae- $\boldsymbol{n}$, and CoF@B-Ph-ae-n (Fig. 2 and Table 1), implying that the physisorption properties of porous polymers can be tuned by introducing the fluoride anion and different metal cations, without changing the backbone of the polymer networks.

Encouraged by the success in the loading of cobalt cations, we further investigated the inclusion of other metal cations in these anionic porous polymers. For instance, nickel(II) acetate or iron(II) nitrate was added to the dispersion of F@B-Ph-ae-3 in THF, followed by stirring for 2 days, respectively. The resulting $\mathrm{Ni}$ or Fe containing porous polymers (NiF@B-Ph-ae-3 and FeF@B-Ph-ae-3) exhibit a loading amount of $1.05 \mathrm{wt} \%$ and 3.11 wt\% respectively. Moreover, the capability of neutral porous

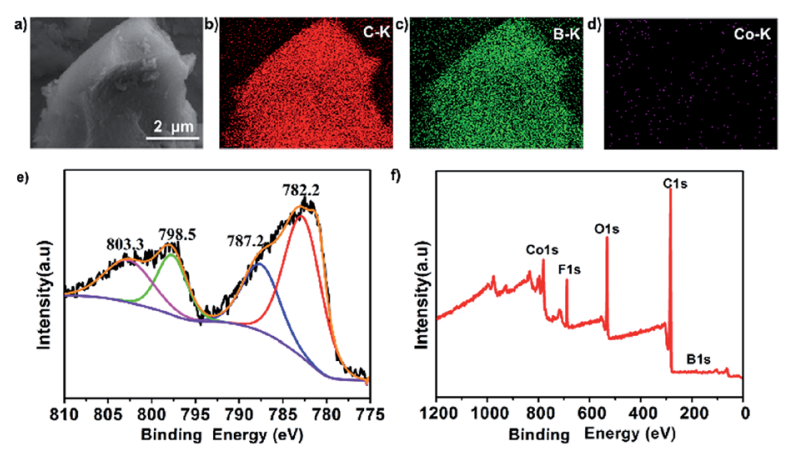

Fig. 3 (a) Typical scanning electron microscopy (SEM) image of CoF@B-Ph-ae-3 and the corresponding elemental mapping images of (b) carbon, (c) boron, and (d) cobalt. The XPS spectra of CoF(aB-Ph-ae3: (e) Co $2 p$ core-level spectrum and (f) the survey spectrum. polymers B-Ph-ae-n to selectively capture metal ions from a mixture system was further examined (Fig. S13†). Reasonably, the ratios of the transition metals loaded in an anionic sample were governed by the corresponding ion exchange constants. ${ }^{31}$ Therefore, the as-synthesized Lewis acid-based neutral porous polymers might have potential application to selectively collect $\mathrm{F}^{-}$and heavy metal ions in the polluted environment through a one-pot process.

It is well known that transition metal ion-loaded porous materials are promising heterogeneous catalysts..$^{33}$ The size and shape selectivity ${ }^{\mathbf{3 a}, 34}$ for a porous catalyst plays a key role in the controlled synthesis of molecules with a certain size or configuration. Thus, the as-prepared MF@B-Ph-ae- $\boldsymbol{n}$ porous polymers, loaded with different transition metal ions, presumably possess heterogeneous catalytic properties highly related to their unique porous structures. With these in our mind, homocoupling reactions of aromatic Grignard reagents catalyzed by employing CoF@B-Ph-ae-n loaded with a stoichiometric amount of cobalt cations (calculated on the basis of the Grignard reagent) were carried out. To elucidate the catalytic activity and size selectivity of CoF@B-Ph-ae- $\boldsymbol{n}$, a series of substrates of different sizes were examined: phenyl Grignard reagent $\left(0.43 \times 0.84 \mathrm{~nm}^{2}\right)$, 3-methylphenyl Grignard reagent $\left(0.47 \times 0.84 \mathrm{~nm}^{2}\right), 4$-methylphenyl Grignard reagent $(0.43 \times$ $\left.0.93 \mathrm{~nm}^{2}\right)$, and 4-ethylphenyl Grignard reagent $(0.43 \times 1.10$ $\left.\mathrm{nm}^{2}\right)$. The homocoupling products were isolated, and the catalytic data are summarized in Table 2. CoF@B-Ph-ae-3, with the largest mesoporous structure, provided the homocoupling products of biphenyl, 4,4'-dimethylbiphenyl, and 3,3'-dimethylbiphenyl in high yields of $91 \%, 84 \%$ and $81 \%$, respectively, but a poor yield of $14 \%$ for $4,4^{\prime}$-diethylbiphenyl with a larger molecular size. While the microporous CoF@B-Ph-ae-2 only resulted in a high conversion of $80 \%$ for biphenyl, but no larger size molecule 4,4'-dimethylbiphenyl or 4,4'-diethylbiphenyl was found and isolated as the product. Interestingly, using 3-methylphenyl Grignard reagent as the substrate, which has a smaller size than 4-methylphenyl Grignard reagent, the microporous CoF@B-Ph-ae-2 enables delivering a remarkable catalytic activity up to a yield of $68 \%$. As a comparison, $\mathrm{CoCl}_{2}$ (its amount was calculated relative to the content of cobalt in

Table 2 Homocoupling reaction of aromatic Grignard reagents by Co(II)-loaded ionic porous polymers ${ }^{a}$

$\begin{array}{lllll}\text { Reactant } & & & \\ \text { Product } & & & \end{array}$

${ }^{a}$ Reaction conditions: $1.6 \mathrm{mmol}$ Grignard reagents, CoF@B-Ph-ae- $\boldsymbol{n}$ containing $0.8 \mathrm{mmol}$ cobalt, $10 \mathrm{~mL}$ of THF at $55{ }^{\circ} \mathrm{C}, 1 \mathrm{~h} .{ }^{b}$ Isolated yield after chromatographic purification. ${ }^{32}$ 
CoF@B-Ph-ae-n) was used as a catalyst for the homocoupling of the aromatic Grignard reagents under the same reaction conditions, which offered the coupling products biphenyl, 4,4'-dimethylbiphenyl, 3,3'-dimethylbiphenyl or 4,4'-diethylbiphenyl in good yields without efficient size selectivity. Therefore, the porous structures had the major impact on the catalytic behavior through spatially controlling the formation of the species in the process of homocoupling reactions (e.g. the intermediates formed in the transmetallation step).

To further probe the catalytic activities and selectivities with respect to the porous structures, a mixture containing phenyl, 4-methylphenyl, and 4-ethylphenyl Grignard reagents in $1: 1: 1$ eq. was treated with CoF@B-Ph-ae-n containing a stoichiometric amount of cobalt cations in THF under mild conditions $\left(55{ }^{\circ} \mathrm{C}\right)$. Gas chromatography mass-spectrometry (GC-MS) analyses revealed that the distribution of coupling products is highly dependent on the pore size of the as-made anionic porous polymers, and the microporous CoF@B-Ph-ae-2 showed the best size selectivity for the formation of biphenyl homocoupling products (Table S2, Fig. S14-S18†). Undoubtedly, such results will be helpful for developing new catalytic systems with high size and shape selectivity.

\section{Conclusions}

In conclusion, we have developed a series of Lewis acid boroncontaining conjugated polymers with hierarchical porous structures via typical transition metal-catalyzed cross-coupling reactions. The distinct capability of the resulting neutral porous polymers to selectively capture fluoride ions provides a highefficiency conversion into stable anionic porous polymers. Importantly, the fluoride anion binding boron in a solid sample, for the first time, was clearly characterized through solid-state ${ }^{11} \mathrm{~B}$ MAS NMR spectroscopy, which would provide a very valuable reference for the corresponding investigation. Upon a simple ion-exchange process, various heavy metal cations were able to be loaded into the networks of the anionic porous polymers. Generally, such a synthetic strategy might represent an efficient approach to versatile functional porous materials via a successful loading process. As an example, the cobalt(II)-loaded porous polymers exhibit a distinct size selectivity for homocoupling reactions of aryl Grignard reagents, which is highly related to the porous structures. Using such a simple ion exchange strategy, various catalytically active metal ions can be rationally incorporated into the as-prepared anionic porous polymers, resulting in new heterogeneous catalytic systems. One can expect that the catalytic behavior is tunable not only by the pore structures of the porous polymers, but also through introduction of other ligands by a "ship in bottle" technique. Moreover, simultaneous integration of multi-metals into the porous polymers may provide synergetic effects for certain catalytic organic transformations with the size and shape control. On the other hand, the pronounced capability to selectively recycle and reuse environmentally harmful fluoride anions and heavy metals makes the as-prepared boron-based porous polymers promising candidates for potential applications in the comprehensive management of the environment.

\section{Acknowledgements}

This work was financially supported by the National Basic Research Program of China (973 Program: 2013CBA01602 and 2012CB933404), the Natural Science Foundation of China (21574080, 51403126 and 21102091), the Shanghai Committee of Science and Technology (15JC1490500) and an ERC grant on 2DMATER. We also thank the Instrumental Analysis Center of Shanghai Jiao Tong University for providing some measurements.

\section{Notes and references}

1 A. G. Slater and A. I. Cooper, Science, 2015, 348, 8075.

2 (a) X. Zhu, C. Tian, S. M. Mahurin, S.-H. Chai, C. Wang, S. Brown, G. M. Veith, H. Luo, H. Liu and S. Dai, J. Am. Chem. Soc., 2012, 134, 10478; (b) J. Zhang, S. H. Chai, Z. A. Qiao, S. M. Mahurin, J. Chen, Y. Fang, S. Wan, K. Nelson, P. Zhang and S. Dai, Angew. Chem., Int. Ed., 2015, 54, 932.

3 (a) Q. Fang, S. Gu, J. Zheng, Z. Zhuang, S. Qiu and Y. Yan, Angew. Chem., Int. Ed., 2014, 53, 2878; (b) R. Palkovits, M. Antonietti, P. Kuhn, A. Thomas and F. Schuth, Angew. Chem., Int. Ed., 2009, 48, 6909; (c) H. Fei, J. Shin, Y. S. Meng, M. Adelhardt, J. Sutter, K. Meyer and S. M. Cohen, J. Am. Chem. Soc., 2014, 136, 4965.

4 (a) F. Vilela, K. Zhang and M. Antonietti, Energy Environ. Sci., 2012, 5, 7819; (b) S. Jin, H. J. Son, O. K. Farha, G. P. Wiederrecht and J. T. Hupp, J. Am. Chem. Soc., 2013, 135, 955; (c) Y. Kou, Y. Xu, Z. Guo and D. Jiang, Angew. Chem., Int. Ed., 2011, 50, 8753.

5 (a) H. C. Zhou, J. R. Long and O. M. Yaghi, Chem. Rev., 2012, 112, 673; (b) Z. J. Lin, J. Lu, M. Hong and R. Cao, Chem. Soc. Rev., 2014, 43, 5867; (c) Y. Liu, W. Xuan and Y. Cui, Adv. Mater., 2010, 22, 4112; (d) A. Corma, H. García and F. X. L. Xamena, Chem. Rev., 2010, 110, 4606.

6 (a) W. J. Roth, P. Nachtigall, R. E. Morris and J. Cejka, Chem. Rev., 2014, 114, 4807; (b) M. E. Davis, Chem. Mater., 2013, 26, 239; (c) A. Karmakar, A. V. Desai and S. K. Ghosh, Coord. Chem. Rev., 2016, 307, 313.

7 (a) G. Sartori and R. Maggi, Chem. Rev., 2011, 111, 181; (b) P. H. Espeel, B. Janssens and P. A. Jacobs, J. Org. Chem., 1993, 58, 7688.

8 S. Saravanamurugan, M. Paniagua, J. A. Melero and A. Riisager, J. Am. Chem. Soc., 2013, 135, 5246.

9 S. Tontisirin and S. Ernst, Angew. Chem., Int. Ed., 2007, 46, 7304.

10 M. Moliner, F. Rey and A. Corma, Angew. Chem., Int. Ed., 2013, 52, 13880.

11 (a) D. Wu, F. Xu, B. Sun, R. Fu, H. He and K. Matyjaszewski, Chem. Rev., 2012, 112, 3959; (b) R. Dawson, A. I. Cooper and D. J. Adams, Prog. Polym. Sci., 2012, 37, 530; (c) X. Feng, X. Ding and D. Jiang, Chem. Soc. Rev., 2012, 41, 6010; (d) X. M. Liu, Y. W. Zhang, H. Li, S. A, H. Xia and Y. Mu, RSC Adv., 2013, 3, 21267; (e) Q. Sun, Z. Dai, X. Meng and F. S. Xiao, Chem. Soc. Rev., 2015, 44, 6018; $(f)$ C. Pei, T. Ben and S. Qiu, Mater. Horiz., 2015, 2, 11; $(g)$ A. Thomas, Angew. Chem., Int. Ed., 2010, 49, 8328. 
12 Y. Xu, S. Jin, H. Xu, A. Nagai and D. Jiang, Chem. Soc. Rev., 2013, 42, 8012.

13 Y. Yuan, F. Sun, L. Li, P. Cui and G. Zhu, Nat. Commun., 2014, $5,4260$.

14 (a) S. Fischer, J. Schmidt, P. Strauch and A. Thomas, Angew. Chem., Int. Ed., 2013, 52, 12174; (b) Z. Yan, Y. Yuan, Y. Tian, D. Zhang and G. Zhu, Angew. Chem., Int. Ed., 2015, 54, 12733; (c) J. F. V. Humbeck, M. L. Aubrey, A. Alsbaiee, R. Ameloot, G. W. Coates, W. R. Dichtel and J. R. Long, Chem. Sci., 2015, 6, 5499.

15 Y. Liu, X. Xu, F. Zheng and Y. Cui, Angew. Chem., Int. Ed., 2008, 120, 4614.

16 J. X. Jiang, F. Su, A. Trewin, C. D. Wood, H. Niu, J. T. Jones and A. I. Cooper, J. Am. Chem. Soc., 2008, 130, 7710.

17 S. H. Lim, Y. Su and S. M. Cohen, Angew. Chem., Int. Ed., 2012, 51, 5106.

18 (a) M. G. Rabbani and H. M. El-Kaderi, Chem. Mater., 2012, 24, 1511; (b) J.-X. Jiang, F. Su, A. Trewin, C. D. Wood, H. Niu, J. T. A. Jones, Y. Z. Khimyak and A. I. Cooper, J. Am. Chem. Soc., 2008, 130, 7710.

19 (a) W. Lu, Z. Wei, D. Yuan, J. Tian, S. Fordham and H.-C. Zhou, Chem. Mater., 2014, 26, 4589; (b) J. X. Jiang, F. Su, A. Trewin, C. D. Wood, N. L. Campbell, H. Niu, C. Dickinson, A. Y. Ganin, M. J. Rosseinsky, Y. Z. Khimyak and A. I. Cooper, Angew. Chem., Int. Ed., 2007, 46, 8574; (c) X. Zhuang, F. Zhang, D. Wu, N. Forler, H. Liang, M. Wagner, D. Gehrig, M. R. Hansen, F. Laquai and X. Feng, Angew. Chem., Int. Ed., 2013, 52, 9668; (d) X. Zhuang, F. Zhang, D. Wu and X. Feng, Adv. Mater., 2014, 26, 3081; (e) X. Zhuang, D. Gehrig, N. Forler, H. Liang, M. Wagner, M. R. Hansen, F. Laquai, F. Zhang and X. Feng, Adv. Mater., 2015, 27, 3789; (f) Y. Su, Z. Yao, F. Zhang, H. Wang, Z. Mics, E. Cánovas, M. Bonn, X. Zhuang and X. Feng, Adv. Funct. Mater., 2016, 26, 5893; (g) K. Yuan, X. Zhuang, H. Fu, G. Brunklaus, M. Forster, Y. Chen, X. Feng and U. Scherf, Angew. Chem., Int. Ed., 2016, 55, 6858.

20 (a) S. Brunauer, L. S. Deming, W. E. Deming and E. Teller, J. Am. Chem. Soc., 1940, 62, 1723; (b) P. B. Balbuena and K. E. Gubbins, Langmuir, 1993, 9, 1801.

$21 \mathrm{~J}$. Weber, J. Schmidt, A. Thomas and W. Bohlmann, Langmuir, 2010, 26, 15650.

22 (a) F. Cheng, E. M. Bonder and F. Jäkle, J. Am. Chem. Soc., 2013, 135, 17286; (b) H. Pan, G.-L. Fu, Y.-H. Zhao and C.-H. Zhao, Org. Lett., 2011, 13, 4830; (c) X. Y. Liu, D. R. Bai and S. Wang, Angew. Chem., Int. Ed., 2006, 45,
5475; (d) W. M. Wan, F. Cheng and F. Jäkle, Angew. Chem., Int. Ed., 2014, 53, 8934.

23 (a) L. Ci, L. Song, C. Jin, D. Jariwala, D. Wu, Y. Li and P. M. Ajayan, Nat. Mater., 2010, 9, 430; (b) H. Li, J. Xing, Z. Xia and J. Chen, Electrochim. Acta, 2014, 139, 331.

24 (a) S. Yamaguchi, S. Akiyama and K. Tamao, J. Am. Chem. Soc., 2001, 123, 11372; (b) Y. Kubo, M. Yamamoto, M. Ikeda, M. Takeuchi, S. Shinkai, S. Yamaguchi and K. Tamao, Angew. Chem., Int. Ed., 2003, 115, 2082; (c) X. Y. Liu, D. R. Bai and S. Wang, Angew. Chem., Int. Ed., 2006, 118, 5601.

25 (a) P. Chen and F. Jäkle, J. Am. Chem. Soc., 2011, 133, 20142; (b) Z. M. Hudson, X.-Y. Liu and S. Wang, Org. Lett., 2010, 13, 300.

26 S. Sole and F. P. Gabbai, Chem. Commun., 2004, 1284.

27 X. D. Hou and B. T. Jones, Encyclopedia of Analytical Chemistry, Wiley Online Library, 2000, p. 9468.

28 K. Maeda, M. Higashi, D. Lu, R. Abe and K. Domen, J. Am. Chem. Soc., 2010, 132, 5858.

29 E. Quartapelle Procopio, F. Linares, C. Montoro, V. Colombo, A. Maspero, E. Barea and J. A. R. Navarro, Angew. Chem., Int. Ed., 2010, 49, 7308.

30 (a) G. Yang, D. Gao, Z. Shi, Z. Zhang, J. Zhang, J. Zhang and D. Xue, J. Phys. Chem. C, 2010, 114, 21989; (b) C. MorenoCastilla, F. J. Maldonado-Hódar and A. F. Pérez-Cadenas, Langmuir, 2003, 19, 5650.

31 (a) W. Meng, H. Li, Z. Xu, S. Du, Y. Li, Y. Zhu, Y. Han, H. Hou, Y. Fan and M. Tang, Chem.-Eur.J., 2014, 20, 2945; (b) S. Kocaoba, Energy Sci. Eng., 2008, 25, 697.

32 The molecular length was calculated by density functional theory (RB3LYP/6-311G** (d,p) level).

33 (a) D. Merki, H. Vrubel, L. Rovelli, S. Fierro and X. Hu, Chem. Sci., 2012, 3, 2515; (b) A. Nag, D. S. Chung, D. S. Dolzhnikov, N. M. Dimitrijevic, S. Chattopadhyay, T. Shibata and D. V. Talapin, J. Am. Chem. Soc., 2012, 134, 13604; (c) P. Kaur, J. T. Hupp and S. T. Nguyen, ACS Catal., 2011, 1, 819; (d) J. Schmidt, J. Weber, J. D. Epping, M. Antonietti and A. Thomas, Adv. Mater., 2009, 21, 702.

34 (a) M. Shakeri, R. J. Klein Gebbink, P. E. de Jongh and K. P. de Jong, Angew. Chem., Int. Ed., 2013, 52, 10854; (b) D. Lesthaeghe, B. De Sterck, V. Van Speybroeck, G. B. Marin and M. Waroquier, Angew. Chem., Int. Ed., 2007, 46, 1311; (c) L. Li, R. Matsuda, I. Tanaka, H. Sato, P. Kanoo, H. J. Jeon, M. L. Foo, A. Wakamiya, Y. Murata and S. Kitagawa, J. Am. Chem. Soc., 2014, 136, 7543; (d) L. Chen, Y. Yang and D. Jiang, J. Am. Chem. Soc., 2010, 132, 9138. 